\title{
Author Index Vol. 94,1995
}

Vol. 94, Supplement 1, has its own Author Index

Abdelaal, A.F. 152 Abe,M. 90 Abellán,M.T. 10 Advani,S.H. 216 Ahmed, M.A.M. 114 Ahmed, S. 7 Alakavuklar, M.N. 210 Al-Ali,A.K. 114 Al-Bashir,A. 114 Al-Fadel Saleh, M. 114 AlKassimi, F.A. 135 Al-Majed, S.A. 135 Al-Mohareb, F. 128 Al-Momen, A.K. 128,135 Altès,A. 10 Al-Zeer,A. 135 Amiot,L. 204 Antinori, A. 163 Anwar, M. 7 Aoki,K. 199 Argilés,B. 69 Asou,N. 32 Astara, G. 48 Atsumi,T. 90 Avila,A. 10

Baaqil,H. 135 Badell,I. 69 Barozzi,P. 169 Barzaghi, A. 44 Bellini, F. 84 Benedetti,D. 214 Bharucha,H. 98 Blaha,M. 167 Bonner, J.A. 59 Bordes, R. 69 Bovo, G. 44 Brunei, S. 69

Bucalossi, A. 58 Budde, R. 74 Burton, I.E. 196

Caenazzo, A. 16 Castagneto, M. 44 Caulet-Maugendre, S. 204 Ceccherini-Nelli, L. 169

Cehreli,C. 210 Chang, J.-Y. 148 Chao,T.-Y. 148 Chen,M.G. 59 Colombini, A. 44 Conter, V. 84 D’Angelo,P. 84 DeLuca, A. 163 DeToni, S. 16 Deutsch,S.I. 214 Diez,R.A. 214 Dispensa, E. 58 Domingo-Allbós, A. 69 Donohue,J.H. 59 Drahosova, M. 167 Drenou,B. 78,204 Dunn, P. 105 Durr,E.D. 59

Earle,J.D. 59 Eisterer,W. 109 El-Hazmi, M.A.F. 128 Esu, S. 48

Fardel,_O. 78 Fauchet,R. 78,204 Fembacher, P.M. 36 Ferreli,A. 48 Figueiredo, M.S. 123

Fischer, R. 173 Fontcuberta, J. 10 Fujisaku, A. 90

Galieni,P. 58 Gaun,S. 199 Goasguen, J. 204 Goh,Y.T. 182 Gopal,R. 216 Grill, J.P. 59 Grulois, I. 78

Habermann, T.M. 59 Harakati,M. 128 Hashino, S. 52 Hatta,Y. 39 Hedge, R.M. 216 Hellerich,U. 74 Higashi,A. 192 Higuchi,S. 192 Hilbe,W. 109 Hino,M. 112 Hirashima,M. 192 Hiromura, K. 1 Hirose,M. 102 Ho,Y.-S. 105 Horie,T. 39 Huisman, T.H.J. 144 Hull,D. 98 Huraib,S. 128

Im,T. 112 Imamura, M. 52 Inada,T. 95 Inamdar, N.A. 216 Intebi,A.D. 214 Irie,K. 23 Ishii,E. 23 Itoyama, T. 156 Iwata, T. 39

Jebavy, L. 167 Jiang, X. 1 Jones, F.G.C. 98

Kambal,A.M. 135 Kanai,H. 1 Kandaswamy, S. 128 Karkhanis, V.C. 216 Kato,K. 90 Kaufmann, R. 173 Kawaguchi, T. 32 Kawakita, M. 32 Kawano, K. 55 Kaya,H. 142 Khan,D.A. 7 Kishikawa, M. 156 Kitano,K. 199 Kiyosawa, K. 199 Kobayashi,H. 52,199 Kobayashi,M. 139 Kobayashi, S. 52 Kodera,Y. 117 Kojima, S. 117 Konwalinka, G. 109 Krejsek, J. 167 Kubuki,Y. 55 Kunieda,Y. 52 Kuroda,Y. 102 Kurosawa, S. 90

Lampis, B. 48 Lamy,T. 78 Lee,L.H. 182 Leonova, J.Y. 144 Le Prise,P.Y. 78,204 Levy, N. 144

Lim,L.C. 182 Linn, Y.C. 182 Luppi,M. 169

Macciò, A. 48 McCluggage, W.G. 98 Macera,M.J. 152

Makimoto, A. 102 Maly,J. 167 Manganini, C. 84 Manteiga, R. 69 Mantovani, G. 48 Marasca, R. 169 Marotta, G. 58 Marra,R. 163 Martenson, J.A. 59 Marti-Vilalta, J.L. 10 Martino,R. 69

Masera, G. 84 Masuyama, T. 23 Matano, S. 142 Mateo, J. 10 Matsuda, I. 192 Matsuda, T. 142 Matsuoka, H. 55 Matsuyama, T. 117 Melichar,B. 167 Mencarini, P. 163 Miyake,H. 32 Miyazaki, S. 23 Mori,S. 32 Motokura,T. 199 Motoyoshi, K. 23 Moussa,K,M. 196 Mura,E. 48 Mura,R. 84 
Nakamura, H. 156 Nakamura, S. 142 Nakano, H. 139 Naoe,H. 192 Narita,A. 199 Nishikawa, K. 32 Nomiyama, M. 23 Nunoi,H. 192

Ohshima,T. 39 Ohtake, S. 142 Okafuji,K. 142 Okamoto,T. 102 Özsoylu,S. 218

Pagano, L. 163 Parikh,P.M. 216 Park, LA. 173

Park,K. 112 Payzin,B. 210 Pietrogrande, F. 16 Piva,E. 16 Plebani,M. 16 Pradella, M. 16

Qaw,F.S. 114 Qureshi, T.Z. 7

Raste,A.S. 216 Reinisch,N. 109 Rizzari,C. 84 Rossi, M.R. 44 Rovelli,A. 44

Sadamori, N. 156 Saito,H. 199 Saito, S. 23 Saleem, M. 7 Sanchez, I. 69 Sanders, P.A. 196 Santa Cruz, G. 48 Sasagawa, I. 156 Sastry, P.S.R.K. 216 Sawamura, M. 1 Schmitz,B. 173 Schoengen, A. 36 Schulz,P.C. 36 Shih,L.-Y. 105 Shimamoto, Y. 139 Shirono,K. 95 Silver,R.T. 152

Silvestri,D. 84 Simões,B.P. 123 Siroky,O. 167 Smetanina, N.S. 144 Spötl,L. 109 Strickler, J.G. 59 Subirá,M. 69 Suga,K. 139 Sureda,A. 69 Suzuki, M. 55 Suzushima, H. 32

Taguchi,Y. 102 Takatsuki, K. 32 Takeuchi, J. 39

Tamburrini, E. 163 Tan, P. 182 Tanaka,N. 142 Tas,P. 204 Tatsumi,N. 112 Teoh,G 182

Thalang,O.N. 112 Thaler, J. 109 Thiele,J. 173 Tien,H.-F. 105 Tien,S.L. 182 Tomonaga, M. 156

Tone,L.G. 123 Torelli,G 169 Trovato,R. 169 Tsao,T.-Y. 148 Tsubouchi, H. 55 Tsuda,H. 95

Tsuda, K. 55 Tsuji,N. 32 Tsukasaki, K. 156

Uchimaru,K. 199 Uderzo,C. 44 Undar,B. 210 Uno,H. 55 Usai,P. 48

Valsecchi, M.G. 84 Verma,R.S. 152

Warsy,A.S. 128 Watanabe,M. 199

Yamada,T. 102 Yamaguchi, H. 23 Yamane, T. 112 Yamashita, K. 55 Yano,S. 1 Yilmaz,U. 210

Yonemura, Y. 32 Yoshida,T. 142 Yu,C.-Y. 148

Zabernigg, A. 109 Zago,M.A. 123

222

Author Index Vol. 94, 1995 